\title{
Supraventricular Tachycardia by Concealed Bypass Tract
}

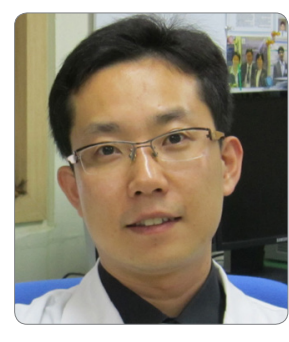

이기홍

전남대학교 의과대학 내과학교실

Ki Hong Lee, MD, PhD

Cardiovascular Medicine, The Heart Center of Chonnam National University Hospital, Republic of Korea

Received: June 21, 2016

Revision Received: November 3, 2016

Accepted: January 4, 2017

Correspondence: Ki Hong Lee, MD, PhD

Cardiovascular Medicine, The Heart Center of

Chonnam National University Hospital, 42,

Jebong-ro, Dong-gu, Gwangju 61469, Republic of

Korea

Tel: +82-62-220-6246, Fax: +82-62-223-3105

E-mail: drgood2@naver.com

Copyright (C) 2017 The Official Journal of Korean Heart

\begin{abstract}
Concealed bypass tract (CBT) results from incomplete development of the atrioventricular (AV) annulus. CBT conducts only in a retrograde direction, and therefore does not cause pre-excitation on standard electrocardiograms. The most common tachycardia associated with $\mathrm{CBT}$ is an orthodromic atrioventricular reentrant tachycardia (AVRT): a pathway involving anterograde circuitry through the AV node and His Purkinje system and retrograde conduction over the accessory pathway. Orthodromic AVRT accounts for approximately $90 \%-95 \%$ cases of AVRT. Most incidences of CBT occur at the left free wall. Vagal maneuvers and/or intravenous (IV) adenosine are recommended for first line acute management of AVRT. However, pharmacological therapy with IV diltiazem, verapamil, or beta blockers can also be effective for acute treatment for orthodromic AVRT in patients who do not show pre-excitation on their resting ECG during sinus rhythm. The first-line ongoing therapy for AVRT is catheter ablation of CBT; when catheter ablation is not indicated or preferred, oral beta blockers, diltiazem, verapamil, flecainide, propafenone, or amiodarone are recommended.
\end{abstract}

Key Words: - Supraventricular Tachycardia -AVNRT

\section{서론}

방실우회로는 방실륜(AV annulus)의 불완전한 발생에 의해 생성되어 방실결절에 비해 빠른 전도속도를 특징으로 하는 비정상 심근세포군이다. 방실우회로는 주로 심방과 심실 사이의 방실륜(AV annulus)에 존재한다. 좌측벽(50\%)에 가장 많이 존재하고, 후중격(20-30\%), 우자유벽(10-20\%), 전중격 (5-10\%)순으로 존재한다고 알려져 있다. 방실우회로는 현성
또는 불현성으로 구분할 수 있고, 전향적, 후향적 또는 양방향으로 전도가 가능하다. 이 중에서 불현성 방실우회로 (concealed bypass tract, $\mathrm{CBT}$ )는 후향적 전도만이 가능하므로, 심전도에서 조기 흥분(pre-excitation)이 관찰되지 않는다. ${ }^{1}$ 본 종설에서는 불현성 방실우회로로 유발될 수 있는 심실상 빈맥의 종류와 기전, 급성기 및 유지 치료 방법에 대해 알아보고자 한다. 


\section{방실 회귀성 빈맥의 병태생리}

방실우회로는 정상 전도계와 순환 회로를 구성하여 다양한 종류의 상심실성 빈맥을 유발한다. 가장 흔히 발생하는 빈맥은 정방향성 방실 회귀성 빈맥(orthodromic atrioventricular reentrant tachycardia, AVRT)이다. 정방향성 방실 회귀성 빈맥은 전도계가 방실결절과 히스속을 이용한 방실전도계를 타고 심실까지 내려가 반대방향으로 방실우회로를 타고 올라와서 다시 심방과 방실결절까지 전도되어 회귀 회로를 완성한다. 정방향성 방실 회귀성 빈맥은 전체 방실 회귀성 빈맥 빈도의 90\%-95\%를 차지하며, 발작성 심실상 빈맥의 약 $35 \%$ 를 차지한다. 일반적으로 방실우회로는 전향적 전도가 불가능하므로 조기 흥분을 유발시키지 않는다. 그런데 조기 흥분이 보이지 않는다고 해서 전부다 전향적 전도가 불가능한 방실우회로로 간주해서는 안된다. 원래 양방향 전도가 가능한 방실우회로임에도 불구하고 간헐적으로 조기 흥분이 보이지 않아 마치 후향적 전도만이 가능한 방실우회로로 보일 수 있기 때문이다. 이러한 성격을 가진 방실우회로는 전체 방실 회귀성 빈맥의 약 $5 \%$ 정도를 차지하는 역방향성 방실 회귀성 빈맥(antidromic AVRT)을 유발할 수 있다. 역방향성 방실 회귀성 빈맥은 전도계가 심방에서 심실로 방실우회로를 타고 내려가서 방실결절을 타고 올라와 회귀 회로를 완성한다.

전방향성 방실 회귀성 빈맥은 심방과 심실이 순차적으로 탈분극되고 정상 방실전도계를 통한 전도속도가 방실우회로를 통한 전도 속도보다 느리기 때문에 $\mathrm{PR}$ 간격이 $\mathrm{RP}$ 간격보다 길어 P파가 QRS파 뒤에 나오게 된다. 역방향성 방실 회귀성 빈맥은 심실의 탈분극이 방실우회로를 타고 내려간 자극에 의해 비정상적으로 전파되기 때문에 심실빈맥에서처럼 QRS 파의 폭이 넓어지게 된다.

대부분의 방실 우회로는 심근과 비슷한 전도 특성을 지니므로 감쇠전도(decremental property)를 보이지 않는다. 그런데 방실우회로가 우중격 부위에 존재할 경우 후향적 감쇠전도를 보일 수 있는 전방향성 방실 회귀성 빈맥을 유발할 수 있고, 이를 지속성 접합부 회귀성 빈맥(permanent junctional reentrant tachycardia, PJRT)이라고 한다. 이 빈맥은 특징적으로 하벽유도 심전도(II, III, aVF)에서 깊게 반전된 후향적 $\mathrm{P}$ 파를 보이고, 우회로의 점감 특성 (diminishing characteristic)으로 인해 긴 RP 간격을 보인다. 또 다른 비전형적 방실우회로는 심방섬유속 (atriofascicular fiber; Mahaim fiber)이다. 심방섬유속은 우심방과 우각의 원위 섬유속을 연결하고 전향적 감쇠전도를 보이지만 후향적 전도는 불가능하다. 심방섬유속에 의해 발생한 방실 회귀성 빈맥은 좌각차단 형태를 보이며, 방실우회로를 통하여 전향적으로 전도되고 방실결절과 히스속을 이용하여 후향적으로 전도되어 회귀 회로를 완성한다. 드문 방실우회로인 결절섬유속(nodofascicular pathway) 회로는 방실결절과 심근을 연결하여 방실 회귀성 빈맥을 유발한다. 드물게 섬유속과 우각 또는 좌각의 근위부와 연결하는 방실우회로가 보고되기는 하였으나 빈맥을 유발하지는 않는다고 알려져 있다.

\section{방실 회귀성 빈맥의 급성기 치료 요법}

방실 회귀성 빈맥 환자의 상태가 위급하지 않다면 미주신경 항진술(vagal maneuver)과 adenosine 정맥 투여를 첫 번째로 시행할 수 있다(class of recommendation I, level of evidence B). 미주신경항진술은 발살바 수기(Valsalva maneuver)와 경정맥동 마사지(carotid sinus massage)가 있으며 신속하게 시행할 수 있다. 발살바 수기는 반드시 환자가 누운 상태에서 시행해야 하고 숨을 참으면서 성문 (glottis)를 10-30초간 막아 흥강 내 압력을 30-40 mmHg 정도 올린다. ${ }^{23}$ 경정맥동 마사지는 경동맥에서 혈관 잡음이 들리지 않는 경우에 시행해야 하고 좌측이나 우측 경정맥동을 5-10초간 지긋이 누른다. ${ }^{2-4}$

Adenosine은 방실 회귀성 빈맥을 90-95\% 정도 종료시키는 효과적인 치료 방법이다(class of recommendation I, level of evidence B) ${ }^{5,6}$ Adenosine은 심방세동을 유발시킬 수 있고 또한 심방세동은 방실우회로를 통하여 심실로 빠르게 전달되어 심실세동을 유발시킬 수 있으므로 반드시 직류충격기가 준비된 상태에서 주사해야 한다.

미주신경항진술을 시행할 수 없거나 adenosine 정맥 투여에 반응이 없으면서 혈역학적으로 불안정할 경우 직류충격 심율동 전환을 시행한다(class of recommendation I, level of evidence B). ${ }^{7}$ 또한 다른 약물치료에 반응이 없거나 금기이면서 혈역학적으로 불안정하다면 직류충격 심율동 전환을 시행한다(class of recommendation I, level of evidence B). 8.9

혈역학적으로 안정되어 있다면 diltiazem, verapamil(class 
of recommendation IIa, level of evidence B), ${ }^{5,10-12}$ 베타차단제(class of recommendation Ila, level of evidence C) $)^{13}$ 등의 약물을 정맥 투여할 수 있다. 이러한 약제들은 평상시 심전도에서 조기흥분이 없는 정방향성 방실 회귀성 빈맥의 종료에 효과적이다(Figure 1).

\section{방실 회귀성 빈맥의 유지 요법}

방실 회귀성 빈맥의 근본적 치료이자 첫 번째 치료는 전극도자절제술이다(class of recommendation I, level of evidence B). ${ }^{14,15}$ 전극도자절제술의 성공율은 93-95\%로 보고되며 약 $3 \%$ 의 환자에서 주요 부작용이 발생한다고 알려져 있다.

전극도자절제술을 시행할 수 없거나 추천되지 않는 경우 경구 베타차단제, diltiazem, vepramil이 방실 회귀성 빈맥 예방 약제로 추천된다(class of recommendation I, level of evidence C). ${ }^{16,17}$ 만약 구조적 심질환이나 허혈성 심질환의 과거력이 없다면 경구 flecainide와 propafenone이
대체약제로 추천된다(class of recommendation Ila, level of evidence B). ${ }^{18,19}$ Flecainide와 propafenone은 방실우회로의 전도를 늦추거나 중단시켜 방실 회귀성 빈맥 발생을 예방한다. 만약 구조적 심질환이나 허혈성 심질환의 과거력이 있다면 경구 dofetilide와 sotalol이 추천된다(class of recommendation $\mathrm{Ilb}$, level of evidence B). ${ }^{20}$ 이들 약제의 경우 QT 간격을 연장시키고 torsa de pointes를 유발할 수 있으므로 주기적으로 심전도를 시행해야 한다. 위의 모든 약제에 반응이 없거나 금기이면서 전극도자절제술을 시행할 수 없고 금기인 경우 경구 amiodarone을 사용해 볼 수 있다 (class of recommendation IIb, level of evidence C). ${ }^{2}$ Digoxin도 방실 회귀성 빈맥 예방 목적으로 사용될 수 있지만 효용성이 낮아 최근에 거의 사용되고 있지 않고 있으므로 위의 모든 약제에 반응이 없거나 금기이면서 전극도자절제술을 시행할 수 없고 금기인 경우 사용해 볼 수 있다(class of recommendation IIb, level of evidence C) (Figure 2). ${ }^{22}$

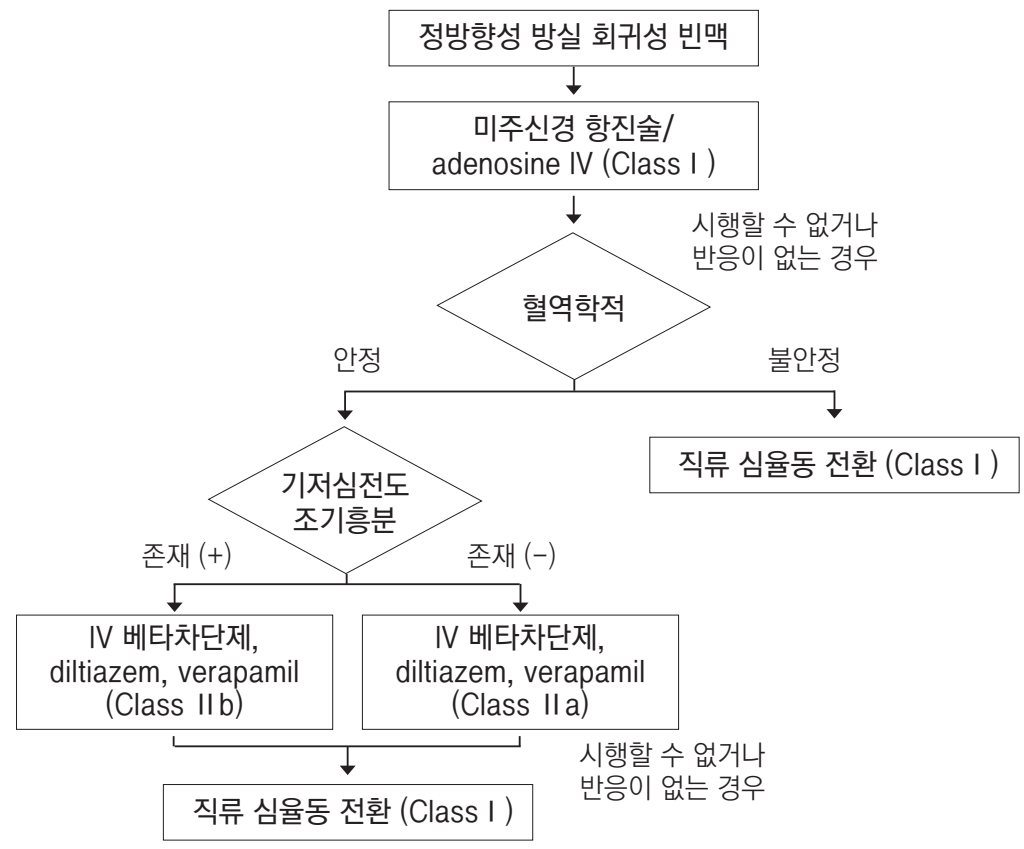

Figure 1. Acute Treatment of Orthodromic AVRT.

AVRT, atrioventricular reentrant tachycardia; IV, intravenous. 


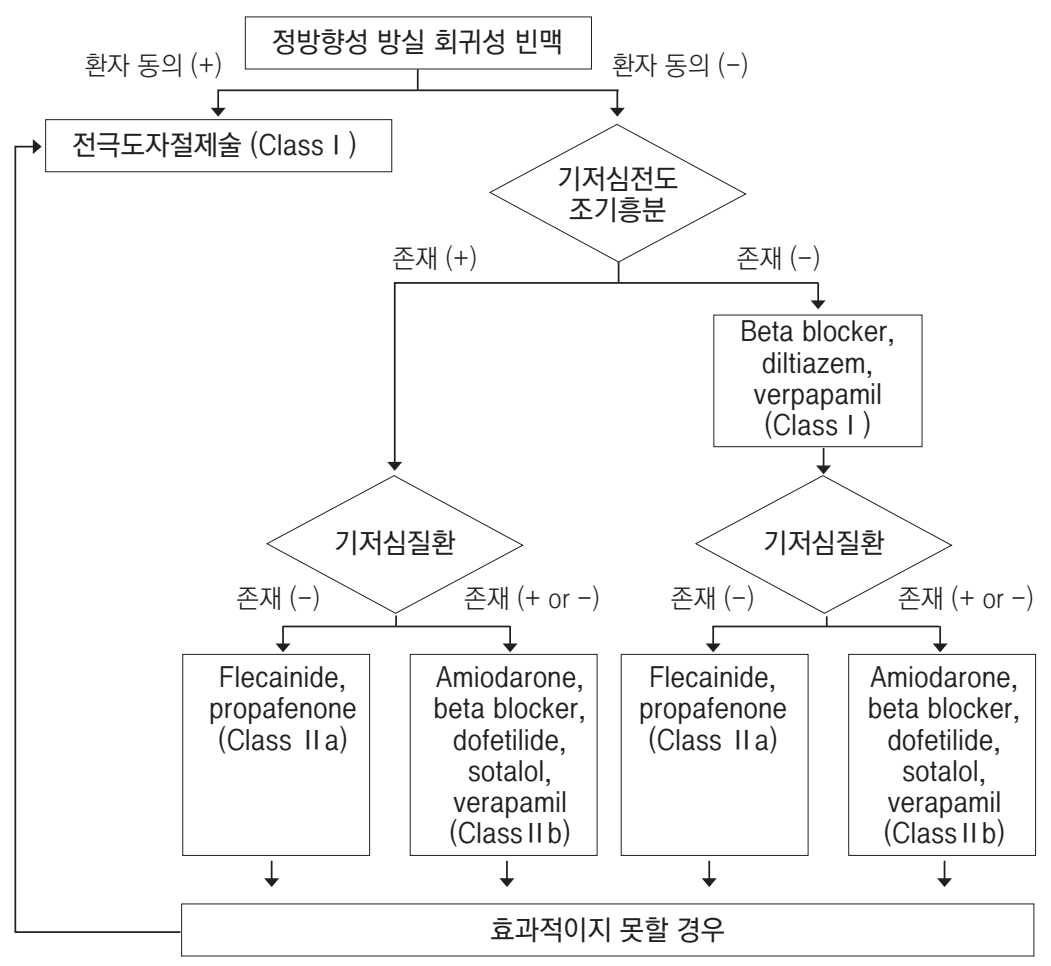

Figure 2. Ongoing management of orthodromic AVRT. AVRT, atrioventricular reentrant tachycardia

\section{References}

1) Page RL, Joglar JA, Caldwell MA, Calkins H, Conti JB, Deal BJ, Estes NA, 3rd, Field ME, Goldberger ZD, Hammill SC, Indik JH, Lindsay BD, Olshansky B, Russo AM, Shen WK, Tracy CM, Al-Khatib SM. 2015 ACC/AHA/HRS guideline for the management of adult patients with supraventricular tachycardia: a report of the American College of Cardiology/American Heart Association task force on clinical practice guidelines and the Heart Rhythm Society. J Am Coll Cardiol. 2016;67:e27-e115.

2) Waxman MB, Wald RW, Sharma AD, Huerta F, Cameron DA. Vagal techniques for termination of paroxysmal supraventricular tachycardia. Am J Cardiol. 1980;46:655-664.

3) Lim SH, Anantharaman V, Teo WS, Goh PP, Tan AT. Comparison of treatment of supraventricular tachycardia by Valsalva maneuver and carotid sinus massage. Ann Emerg Med. 1998;31:30-35.

4) Luber S, Brady WJ, Joyce T, Perron AD. Paroxysmal supraventricular tachycardia: outcome after ED care. Ann Emerg Med. 2001;19:40-42.
5) Delaney B, Loy J, Kelly AM. The relative efficacy of adenosine versus verapamil for the treatment of stable paroxysmal supraventricular tachycardia in adults: A meta-analysis. Eur J Emerg Med. 2011;18:148-152.

6) Furlong R, Gerhardt RT, Farber P, Schrank K, Willig R, Pittaluga J. Intravenous adenosine as first-line prehospital management of narrow-complex tachycardias by ems personnel without direct physician control. Am J Emerg Med. 1995;13:383-388.

7) Neumar RW, Otto CW, Link MS, Kronick SL, Shuster M, Callaway CW, Kudenchuk PJ, Ornato JP, McNally B, Silvers SM, Passman RS, White RD, Hess EP, Tang W, Davis D, Sinz E, Morrison LJ. Part 8: adult advanced cardiovascular life support: 2010 American Heart Association guidelines for cardiopulmonary resuscitation and emergency cardiovascular care. Circulation. 2010;122:S729-S767.

8) Brady WJ, Jr., DeBehnke DJ, Wickman LL, Lindbeck G. Treatment of out-of-hospital supraventricular tachycardia: adenosine vs verapamil. Acad Emerg Med. 1996;3:574-585.

9) Stec S, Krynski T, Kulakowski P. Efficacy of low energy rectilinear 
biphasic cardioversion for regular atrial tachyarrhythmias. Cardiol J. 2011;18:33-38.

10) DiMarco JP, Miles W, Akhtar M, Milstein S, Sharma AD, Platia E, McGovern B, Scheinman MM, Govier WC. Adenosine for paroxysmal supraventricular tachycardia: dose ranging and comparison with verapamil. Assessment in placebo-controlled, multicenter trials. The adenosine for PSVT study group. Ann Intern Med. 1990;113:104-110.

11) Huycke EC, Sung RJ, Dias VC, Milstein S, Hariman RJ, Platia EV. Intravenous diltiazem for termination of reentrant supraventricular tachycardia: a placebo-controlled, randomized, double-blind, multicenter study. J Am Coll Cardiol. 1989;13:538-544.

12) Hamer A, Peter T, Platt M, Mandel WJ. Effects of verapamil on supraventricular tachycardia in patients with overt and concealed Wolff-Parkinson-White syndrome. Am Heart J. 1981;101:600612.

13) Hombach V, Braun V, Hopp HW, Gil-Sanchez D, Behrenbeck DW, Tauchert M, Hilger HH. Antiarrhythmic effects of acute betablockade with atenolol on supraventricular tachycardias at rest and during exercise. Klin Wochenschr. 1981;59:123-133.

14) Calkins H, Yong P, Miller JM, Olshansky B, Carlson M, Saul JP, Huang SK, Liem LB, Klein LS, Moser SA, Bloch DA, Gillette P, Prystowsky E. Catheter ablation of accessory pathways, atrioventricular nodal reentrant tachycardia, and the atrioventricular junction: final results of a prospective, multicenter clinical trial. The Atakr multicenter investigators group. Circulation. 1999;99:262-270.

15) Dagres N, Clague JR, Kottkamp H, Hindricks G, Breithardt G, Borggrefe M. Radiofrequency catheter ablation of accessory pathways. Outcome and use of antiarrhythmic drugs during follow-up. Eur Heart J. 1999;20:1826-1832.
16) Dorian P, Naccarelli GV, Coumel P, Hohnloser SH, Maser MJ. A randomized comparison of flecainide versus verapamil in paroxysmal supraventricular tachycardia. the flecainide multicenter investigators group. Am J Cardiol. 1996;77:89A-95A.

17) Sakurai M, Yasuda H, Kato N, Nomura A, Fujita M, Nishino T, Fujita K, Koike Y, Saito H. Acute and chronic effects of verapamil in patients with paroxysmal supraventricular tachycardia. Am Heart J. 1983;105:619-628.

18) Henthorn RW, Waldo AL, Anderson JL, Gilbert EM, Alpert BL, Bhandari AK, Hawkinson RW, Pritchett EL. Flecainide acetate prevents recurrence of symptomatic paroxysmal supraventricular tachycardia. The flecainide supraventricular tachycardia study group. Circulation. 1991;83:119-125.

19) Chimienti M, Cullen MT, Jr., Casadei G. Safety of flecainide versus propafenone for the long-term management of symptomatic paroxysmal supraventricular tachyarrhythmias. Report from the flecainide and propafenone Italian study (FAPIS) group. Eur Heart J. 1995;16:1943-1951.

20) Cheng CH, Sanders GD, Hlatky MA, Heidenreich P, McDonald KM, Lee BK, Larson MS, Owens DK. Cost-effectiveness of radiofrequency ablation for supraventricular tachycardia. Ann Intern Med. 2000;133:864-876.

21) Feld GK, Nademanee K, Stevenson W, Weiss J, Klitzner T, Singh BN. Clinical and electrophysiologic effects of amiodarone in patients with atrial fibrillation complicating the Wolff-ParkinsonWhite syndrome. Am Heart J. 1988;115:102-107.

22) Bauernfeind RA, Wyndham CR, Dhingra RC, Swiryn SP, Palileo E, Strasberg B, Rosen KM. Serial electrophysiologic testing of multiple drugs in patients with atrioventricular nodal reentrant paroxysmal tachycardia. Circulation. 1980;62:1341-1349. 